\title{
Moriwaki Divisors and the Augmented Base Loci of Divisors on the Moduli Space of Curves
}

\author{
Salvatore Cacciola, Angelo \\ Felice Lopez, \& Filippo Viviani
}

\begin{abstract}
We study the cone of Moriwaki divisors on $\bar{M}_{g}$ by means of augmented base loci. Using a result of Moriwaki, we prove that an $\mathbb{R}$-divisor $D$ satisfies the strict Moriwaki inequalities if and only if $\mathbf{B}_{+}(D) \subseteq \partial \bar{M}_{g}$. Then we draw some interesting consequences on the Zariski decomposition of divisors on $\bar{M}_{g}$, on the minimal model program of $\bar{M}_{g}$, and on the log canonical models $\bar{M}_{g}(\alpha)$.
\end{abstract}

\section{Introduction}

Let $g \geq 3$, and let $\bar{M}_{g}$ be the moduli space of stable curves of genus $g$. A striking result of Gibney, Keel and Morrison [GKM, Thm. 0.9] asserts that any nef divisor on $\bar{M}_{g}$, not linearly equivalent to zero, must be big. In terms of cones of divisors in the Néron-Severi space $N^{1}\left(\bar{M}_{g}\right)_{\mathbb{R}}$, this implies that the nef cone does not meet the boundary of the big cone along rational nonzero classes. As a matter of fact, as we shall see, the same is true for real classes: $\operatorname{Nef}\left(\bar{M}_{g}\right)-\{0\} \subset \operatorname{Big}\left(\bar{M}_{g}\right)$. One way to see this is to consider the Moriwaki cone $\operatorname{Mor}\left(\bar{M}_{g}\right)$, that is, the cone of $\mathbb{R}$-divisors $D$ on $\bar{M}_{g}$ that are nef away from the boundary. The cone $\operatorname{Mor}\left(\bar{M}_{g}\right)$ was explicitly described by Moriwaki [M, Cor. 4.3] in terms of the generators $\lambda, \delta_{0}, \ldots, \delta_{\lfloor g / 2\rfloor}$ : an $\mathbb{R}$-divisor $D \sim a \lambda-b_{0} \delta_{0}-\cdots-b_{\lfloor g / 2\rfloor} \delta_{\lfloor g / 2\rfloor}$ belongs to $\operatorname{Mor}\left(\bar{M}_{g}\right)$ if and only if it is an M-divisor, that is, it satisfies the Moriwaki inequalities

$$
a \geq 0, \quad a \geq \frac{8 g+4}{g} b_{0}, \quad a \geq \frac{2 g+1}{i(g-i)} b_{i} \quad \text { for all } i=1, \ldots,\lfloor g / 2\rfloor .
$$

The starting idea of this paper is that both the Moriwaki cone and its interior, that is, the cone of those $\mathbb{R}$-divisors that satisfy the strict Moriwaki inequalities and that we call strict $M$-divisors, can be interpreted in terms of restricted and augmented base loci.

Received February 20, 2015. Revision received May 9, 2016.

All three authors were partially supported by the MIUR national project "Geometria delle varietà algebriche" PRIN 2010-2011. FV was also supported by the MIUR national project "Spazi di moduli e applicazioni" FIRB 2012, by the Research Network Program GDRE-GRIFGA, and by CMUC—Centro de Matemática da Universidade de Coimbra. 
Recall that the stable base locus $\mathbf{B}(D)$ of an $\mathbb{R}$-Cartier $\mathbb{R}$-divisor $D$ on a normal projective variety $X$ is defined as

$$
\mathbf{B}(D)=\bigcap_{E \geq 0: E \sim \sim_{\mathbb{R}} D} \operatorname{Supp}(E)
$$

with the convention that $\mathbf{B}(D)=X$ if the intersection runs over the empty set.

The augmented base locus and the restricted base locus of $D$ are, respectively,

$$
\mathbf{B}_{+}(D)=\bigcap_{A \text { ample }} \mathbf{B}(D-A) \text { and } \mathbf{B}_{-}(D)=\bigcup_{A \text { ample }} \mathbf{B}(D+A),
$$

where $A$ runs among all ample $\mathbb{R}$-Cartier $\mathbb{R}$-divisors. We have the inclusions $\mathbf{B}_{-}(D) \subseteq \mathbf{B}(D) \subseteq \mathbf{B}_{+}(D)$, and $D$ is big if and only if $\mathbf{B}_{+}(D) \subsetneq X$.

Returning to $\bar{M}_{g}$, the main result of this article, where the assertion on $\mathbf{B}_{-}(D)$ is just a rewriting of $[\mathrm{M}$, Thm. C], is the following:

TheOREM 1 . Let $g \geq 3$, and let $D$ be an $\mathbb{R}$-divisor on $\bar{M}_{g}$. Then

(i) $\mathbf{B}_{-}(D) \subseteq \partial \bar{M}_{g}$ if and only if $D$ is an M-divisor;

(ii) $\mathbf{B}_{+}(D) \subseteq \partial \bar{M}_{g}$ if and only if $D$ is a strict $M$-divisor.

Now nef nonzero divisors are strict M-divisors (see Lemma 3.2); therefore, the first simple consequence of Theorem 1 is that

$$
\operatorname{Nef}\left(\bar{M}_{g}\right)-\{0\} \subset \operatorname{Int}\left(\operatorname{Mor}\left(\bar{M}_{g}\right)\right) \subset \operatorname{Big}\left(\bar{M}_{g}\right) .
$$

Note that this gives another proof on $\bar{M}_{g}$, but for $\mathbb{R}$-divisors, of [GKM, Thm. 0.9].

In Figure 1 we made a schematic picture of the Moriwaki cone and its relative position with respect to the nef cone $\operatorname{Nef}\left(\bar{M}_{g}\right)$ and the pseudoeffective cone $\overline{\operatorname{Eff}}\left(\bar{M}_{g}\right)$ of $\bar{M}_{g}$.

We point out that from (1) it follows that $\operatorname{Mor}\left(\bar{M}_{g}\right)$ is a simplicial polyhedral cone whose extremal rays are generated by the boundary divisors $\left\{\delta_{0}, \ldots, \delta_{\lfloor g / 2\rfloor}\right\}$ and by the Moriwaki divisor

$$
M=(8 g+4) \lambda-g \delta_{0}-\sum_{i=1}^{\lfloor g / 2\rfloor} 4 i(g-i) \delta_{i} .
$$

Although we do not use the following facts, $M$ is known to be a big divisor (see [Lo]), and consequently the boundary of $\operatorname{Mor}\left(\bar{M}_{g}\right)$ intersects the boundary of the pseudoeffective cone $\overline{\operatorname{Eff}}\left(\bar{M}_{g}\right)$ only in the common codimension-one face formed by effective boundary divisors. The Moriwaki divisor also appears in the works of Hain-Reed [HR] and Hain [H].

Another consequence of Theorem 1 is that it gives many compactifications of $M_{g}$, generalizing [GKM, Cor. 0.11].

Corollary 1 . Let $g \geq 3$, let $D$ be a $\mathbb{Q}$-divisor on $\bar{M}_{g}$ such that $\kappa\left(\bar{M}_{g}, D\right) \geq$ 0 , and for $m \in \mathbb{N}$, consider the map $\varphi_{m D}: \bar{M}_{g} \rightarrow \mathbb{P} H^{0}\left(\bar{M}_{g}, m D\right)$. If $D$ is a strict $M$-divisor, then there exists $m_{0} \in \mathbb{N}$ such that $\varphi_{m m_{0} D}$ is an isomorphism over $M_{g}$ for any $m \in \mathbb{N}$. Vice versa, if there exists $m_{1} \in \mathbb{N}$ such that $\varphi_{m m_{1} D}$ is an 


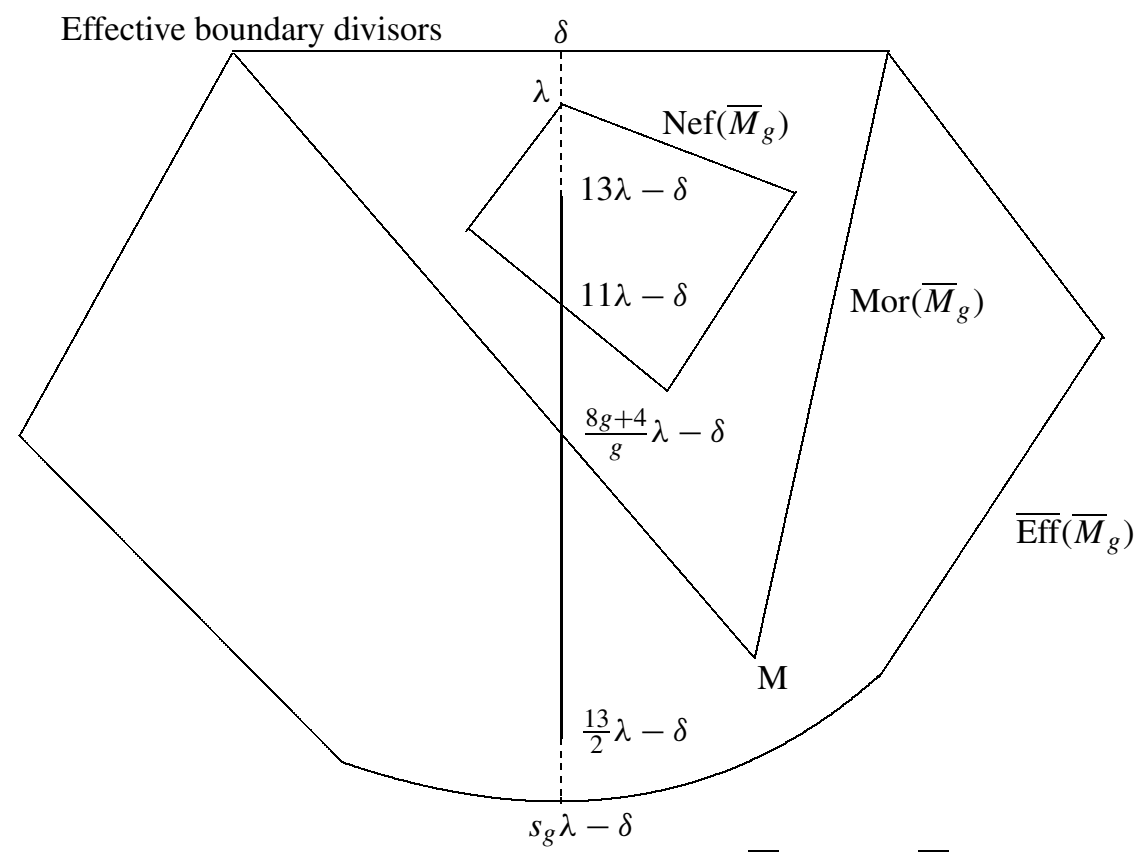

Figure 1 A section of the three cones $\operatorname{Nef}\left(\bar{M}_{g}\right) \subseteq \operatorname{Mor}\left(\bar{M}_{g}\right) \subseteq$ $\overline{\operatorname{Eff}}\left(\bar{M}_{g}\right)$ and their intersection with the plane $\langle\lambda, \delta\rangle$. Here $s_{g}$ is the slope of $\bar{M}_{g}$ (see [HMo]), which, for the sake of the picture, is assumed to be $\leq \frac{13}{2}$ (this is known to be true for $g \geq 22$ )

isomorphism over $M_{g}$ for any $m \in \mathbb{N}$, then $\mathbf{B}_{+}(D) \subseteq \partial \bar{M}_{g} \cup \mathbf{B}(D)$, and $D$ is a strict M-divisor when $\mathbf{B}(D) \subseteq \partial \bar{M}_{g}$.

It would be interesting to know whether some of the compactifications obtained in Corollary 1 arise from (stable) modular compactifications in the sense of [S, Defs. 1.1 and 1.2] or if, conversely, all the (stable) modular compactifications of [S] arise from strict M-divisors.

In another direction, it would be desirable to extend Theorem 1 and Corollary 1 to $\bar{M}_{g, n}$. A first partial result in this direction has been established in [CL, Cor. 2], where it is proved, as a consequence of [GKM, Thm. 0.9], that big and nef divisors on $\bar{M}_{g, n}$ have their augmented base loci contained in the boundary of $\bar{M}_{g, n}$.

We can also apply Theorem 1 to get some information on the log canonical models introduced by Hassett and Hyeon [HH1; HH2],

$$
f_{\alpha}: \bar{M}_{g} \rightarrow \bar{M}_{g}(\alpha)=\operatorname{Proj}\left(\bigoplus_{m \geq 0} H^{0}\left(\bar{M}_{g},\lfloor m(13 \lambda-(2-\alpha) \delta)\rfloor\right)\right)
$$

for $\alpha \in[0,1] \cap \mathbb{Q}$, where $f_{\alpha}$ is the standard rational map associated to the construction of Proj (see [ST, Tag 01NK]), or equivalently, the map associated to the linear system $|m(13 \lambda-(2-\alpha) \delta)|$ for $m \gg 0$ (see Corollary 2.2). 
In Figure 1, we have depicted the intersection of the segment $\left\{\frac{13}{2-\alpha} \lambda-\delta: \alpha \in\right.$ $[0,1]\}$ with the cones $\operatorname{Nef}\left(\bar{M}_{g}\right) \subseteq \operatorname{Mor}\left(\bar{M}_{g}\right) \subseteq \overline{\operatorname{Eff}}\left(\bar{M}_{g}\right)$.

It has been asked by Hassett ${ }^{1}$ whether the map $f_{\alpha}$ is an isomorphism over $M_{g}$ when $\alpha>\frac{3 g+8}{8 g+4}$. We give an affirmative answer in the following:

Corollary 2. Let $g \geq 3$. Then

(i) $f_{\alpha}$ is an isomorphism over $M_{g}$ if and only if $\alpha>\frac{3 g+8}{8 g+4}$;

(ii) If $\alpha=\frac{3 g+8}{8 g+4}$, then $f_{\alpha}$ is defined over $M_{g}$, and it contracts the hyperelliptic locus $\bar{H}_{g} \subset \bar{M}_{g}$;

(iii) If $\alpha<\frac{3 g+8}{8 g+4}$, then the hyperelliptic locus $\bar{H}_{g}$ is contained in $\mathbf{B}_{-}(13 \lambda-(2-$ $\alpha) \delta)$.

Note that part (iii) implies that $f_{\alpha}$ is not defined over $\bar{H}_{g}$ whenever $\bar{H}_{g}$ is not contained in a divisorial component of $\mathbf{B}(13 \lambda-(2-\alpha) \delta$ ) (which of course can occur only for $g \geq 4)$. We also remark that, whenever $13 \lambda-(2-\alpha) \delta$ is big, we have that $\mathbf{B}_{-}(13 \lambda-(2-\alpha) \delta)=\mathbf{B}(13 \lambda-(2-\alpha) \delta)$ (see Remark 3.3).

Our next goal is deduce, from Theorem 1, some interesting consequences on the Zariski decomposition of divisors and on the minimal models of $\bar{M}_{g}$.

Recall that, given a pseudoeffective $\mathbb{R}$-Cartier $\mathbb{R}$-divisor $D$ on a normal projective variety $X$, we say that $D$ has an $\mathbb{R}-C K M$ Zariski decomposition (CKM stands for Cutkoski-Kawamata-Moriwaki) if we can write

$$
D=P+N,
$$

where $P, N$ are $\mathbb{R}$-Cartier $\mathbb{R}$-divisors such that $P$ is nef, $N$ is effective, and $h^{0}(\lfloor m D\rfloor)=h^{0}(\lfloor m P\rfloor)$ for all $m \in \mathbb{N}$, where $\lfloor m D\rfloor$ (and $\lfloor m P\rfloor$ ) is the rounddown.

Whereas on a smooth surface a Zariski decomposition always exists, by the celebrated result of Zariski, in general, on higher-dimensional varieties, divisors may or may not have an $\mathbb{R}-\mathrm{CKM}$ Zariski decomposition, even if we allow to pass to a birational model [N, Thm. IV.2.10]. On the other hand, on a variety of nonnegative Kodaira dimension, the canonical bundle is expected to admit an $\mathbb{R}$ CKM Zariski decomposition, after passing to a birational model, as a consequence of the conjectured existence of minimal models.

On $\bar{M}_{g}$ we obtain the following:

Corollary 3. Let $g \geq 3$, and let $D$ be an $\mathbb{R}$-divisor on $\bar{M}_{g}$ such that $\kappa(D) \geq 1$. If $D$ has an $\mathbb{R}$-CKM Zariski decomposition, then $D$ is a strict $M$-divisor. In particular, when $\kappa\left(\bar{M}_{g}\right) \geq 1$ (currently known for $g \geq 22$ ), the canonical divisor $K_{\bar{M}_{g}}$ does not have an $\mathbb{R}-C K M$ Zariski decomposition.

\footnotetext{
${ }^{1}$ In the open problem session of the AIM workshop "The log minimal model program for the moduli space of curves", Palo Alto (California, USA), 10-14 December 2012. During the same problem session, M. Fedorchuk said that he could answer to the question away from the hyperelliptic locus.
} 
We stress that, for $g \geq 24$ or $g=22$, since $K_{\bar{M}_{g}}$ is known to be big by [HMu; EH; F], the minimal model of $\bar{M}_{g}$ exists by [BCHM, Lemma 10.1 and Thm. 1.2], whence the pull-back of $K_{\bar{M}_{g}}$ does have an $\mathbb{R}$-CKM Zariski decomposition on some birational model of $\bar{M}_{g}$. On the other hand, $\bar{M}_{g}$ is an interesting example of a normal projective variety whose canonical bundle does not have an $\mathbb{R}-\mathrm{CKM}$ Zariski decomposition.

COROLlaRY 4. Let $g$ be such that $\kappa\left(\bar{M}_{g}\right) \geq 1$ (currently known for $g \geq 22$ ). Then there is no $K_{\bar{M}_{g}}$-nonpositive projective birational morphism $f: \bar{M}_{g} \rightarrow X$ onto a normal $\mathbb{Q}$-Gorenstein variety $X$ with $K_{X}$ nef. In particular, if $K_{\bar{M}_{g}}$ is big (currently known for $g \geq 24$ or $g=22)$, consider a rational map $f: \bar{M}_{g} \rightarrow-\left(\bar{M}_{g}\right)_{\min }$ to a minimal model obtained via contractions and flips of $K$-negative extremal rays. Then $f$ cannot be a morphism, that is, it is not possible to reach a minimal model of $\bar{M}_{g}$ only via contractions of extremal rays: at some step, one must flip.

Note that, whenever $K_{\bar{M}_{g}}$ is big, we have that $\mathbf{B}_{-}\left(K_{\bar{M}_{g}}\right)=\mathbf{B}\left(K_{\bar{M}_{g}}\right)$ (see Remark 3.3).

Unless otherwise specified, we work throughout the paper over an algebraically closed field $k$ of characteristic 0 , although we expect that our results should hold in arbitrary characteristic (see Section 3.1).

\section{Generalities on Proj and Zariski Decomposition}

We collect in this section some general facts that will be used in the proofs. They are all most likely well known, but we include them for the lack of a reference (even though a similar version of Lemma 2.1 can be found in [HK, Lemma 1.6]).

Recall that, given a $\mathbb{Q}$-Cartier $\mathbb{Q}$-divisor $D$ on a normal projective variety $X$, its ring of sections is

$$
R(X, D)=\bigoplus_{m \geq 0} H^{0}(X,\lfloor m D\rfloor),
$$

and if $m D$ is Cartier and $H^{0}(X, m D) \neq\{0\}$, then we denote by

$$
\varphi_{m D}: X \rightarrow Y_{m} \subseteq \mathbb{P} H^{0}(X, m D)
$$

the map associated to $|m D|$, where $Y_{m}$ is the closure of its image (endowed with its reduced scheme structure).

LEMma 2.1. Let $X$ be a normal projective variety defined over an algebraically closed field $k$ of arbitrary characteristic, and let $D$ be a $\mathbb{Q}$-Cartier $\mathbb{Q}$-divisor on $X$ such that $\kappa(X, D) \geq 0$ and $R(X, D)$ is a finitely generated $k$-algebra. Then there is $m_{0} \in \mathbb{N}$ such that $Y_{a m_{0}} \cong \operatorname{Proj}(R(X, D))$ is normal for all $a \in \mathbb{N}$. Moreover, with this identification, the standard rational map associated with the construction of Proj (see [ST, Tag 01NK]), $f_{D}: X \rightarrow \operatorname{Proj}(R(X, D))$ coincides with $\varphi_{a m_{0} D}$ : $X \rightarrow Y_{a m_{0}}$ for all $a \in \mathbb{N}$. 
Proof. By [EGA2, Prop. 2.4.7(i)] we can assume that $D$ is Cartier. By [EGA2, Lemma 2.1.6(v)] there exists $s \in \mathbb{N}$ such that

$$
S^{h} H^{0}(X, s D) \rightarrow H^{0}(X, h s D) \quad \text { is surjective for all } h \in \mathbb{N} \text {. }
$$

Since $\kappa(X, D) \geq 0$, we get that $H^{0}(X, s D) \neq\{0\}$ and that $\mathbf{B}(D)=\operatorname{Bs}(|h s D|)$ for all $h \in \mathbb{N}$. Let $p: \widetilde{X} \rightarrow X$ be the normalized blow-up of $X$ along the base ideal of $|s D|$, so that we have the diagram

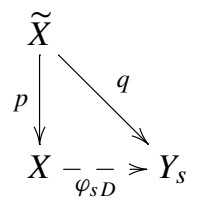

with $\tilde{X}$ normal and $p$ birational. We can write

$$
p^{*}(s D)=M+F
$$

with $|M|$ base-point free, $F$ base component of $\left|p^{*}(s D)\right|$, and $p(\operatorname{Supp}(F))=$ $\mathbf{B}(D)$. Since $X$ is normal and $p$ is birational, we have, by Zariski's main theorem, that $p$ is an algebraic fiber space [La, Def. 2.1.11], and therefore, for all $h \in \mathbb{N}$,

$$
H^{0}(X, h s D) \cong H^{0}\left(\tilde{X}, p^{*}(h s D)\right) \text {. }
$$

It follows by finite generation that, for all $h \in \mathbb{N}, h F$ is the base component of $\left|p^{*}(h s D)\right|$, whence

$$
H^{0}\left(\widetilde{X}, p^{*}(h s D)\right) \cong H^{0}(\widetilde{X}, h M) .
$$

But then $Y_{h s}=\operatorname{Im}\left\{\varphi_{h M}: \widetilde{X} \rightarrow \mathbb{P} H^{0}(\widetilde{X}, h M)\right\}$ for all $h \in \mathbb{N}$. On the other hand, by [La, Thm. 2.1.27] there are $h_{0} \in \mathbb{N}$ and an algebraic fiber space $\phi: \widetilde{X} \rightarrow Z$ such that $\varphi_{h M}=\phi$ and $\operatorname{Im} \varphi_{h M}=Z$ for all $h \geq h_{0}$. Now $Z$ is normal by [La, Thm. 2.1.15], whence setting $m_{0}=h_{0} s$, we get that $Y_{a m_{0}}=Z$ is normal for all $a \in \mathbb{N}$.

Let $A$ be an ample divisor on $Z$ such that $h_{0} M=\phi^{*}(A)$. Since $\phi$ is an algebraic fiber space, we get

$$
H^{0}\left(\tilde{X}, s h_{0} M\right)=H^{0}\left(\tilde{X}, \phi^{*}(s A)\right) \cong H^{0}(Z, s A) .
$$

Since the product in a ring of sections is given by multiplication of sections, we deduce by (5), (6), and (7) that $R\left(X, m_{0} D\right) \cong R\left(\widetilde{X}, p^{*}\left(m_{0} D\right)\right) \cong$ $R\left(\widetilde{X}, h_{0} M\right) \cong R\left(\widetilde{X}, \phi^{*}(A)\right) \cong R(Z, A)$. Finally, by [EGA2, Prop. 2.4.7(i)] we get $\operatorname{Proj}(R(X, D)) \cong \operatorname{Proj}\left(R\left(X, m_{0} D\right)\right) \cong \operatorname{Proj}(R(Z, A)) \cong Z$ since $A$ is ample.

By [ST, Tag $01 \mathrm{NK}$ ], given a graded ring $S$, a scheme $T$ with a line bundle $\mathcal{L}$, and a homomorphism of graded rings $\psi: S \rightarrow R(T, \mathcal{L})$, there is a morphism

$$
f_{D}: U(\psi) \rightarrow \operatorname{Proj}(R(X, D)),
$$

where $U(\psi)$ is the union of the open subsets $T_{\psi(f)}$ with $f \in S_{d}, d>0$. In our case, setting $T=X, \mathcal{L}=\mathcal{O}_{X}(D), S=R(X, D)$, and $\psi=\operatorname{Id}_{R(X, D)}$, we have that $U(\psi)=X-\mathbf{B}(D)$, and we get a rational map $f_{D}: X \rightarrow \operatorname{Proj}(R(X, D))$ defined on $X-\mathbf{B}(D)$. On the other hand, for any $d \in \mathbb{N}$ such that $\mathbf{B}(D)=\operatorname{Bs}(|d D|)$, by [ST, Tag $01 \mathrm{NK}$ ] we have that $f_{D}$ coincides on $X-\mathbf{B}(D)$ with the morphism 
$X-\mathbf{B}(D) \rightarrow \operatorname{Proj}(R(X, D))$ defined on [ST, Tag01N8], which, given the immersion $\operatorname{Proj}(R(X, D)) \subset \mathbb{P}^{r}, r=h^{0}(X, d D)$, is just the morphism $\varphi_{d D}$.

We draw a consequence on the spaces $\bar{M}_{g}(\alpha)$.

CoRollary 2.2. For every $\alpha \in[0,1] \cap \mathbb{Q}$, we have that $\bar{M}_{g}(\alpha)$ is normal and the rational map $f_{\alpha}: \bar{M}_{g} \rightarrow-\bar{M}_{g}(\alpha)$ is given by $\varphi_{m(13 \lambda-(2-\alpha) \delta)}$ for $m$ sufficiently divisible.

Proof. Set $K_{\alpha}=13 \lambda-(2-\alpha) \delta$. We can assume that $\kappa\left(K_{\alpha}\right) \geq 0$. If $\alpha=1$, then the assertion follows by [Mu, Cor. 5.18] (see also [CH, Thm. 1.3]) since $13 \lambda-\delta$ is ample. Now assume that $\alpha<1$ and set $B_{\alpha}=\alpha\left(\Delta_{0}+\Delta_{2}+\cdots+\Delta_{\lfloor g / 2\rfloor}\right)+\frac{\alpha+1}{2} \Delta_{1}$, so that $K_{\alpha}=K_{\bar{M}_{g}}+B_{\alpha}$, and $\left(\bar{M}_{g}, B_{\alpha}\right)$ is klt [KM, Def. 2.34] by [HH1, Proof of Prop. A.13] or [BCHM, Proof of Cor. 1.2.1]. Then $R\left(\bar{M}_{g}, K_{\alpha}\right)$ is a finitely generated $k$-algebra by [BCHM, Cor. 1.1.2], and we just apply Lemma 2.1.

We also need a result about Zariski decompositions.

Lemma 2.3. Let $X$ be a normal $\mathbb{Q}$-factorial projective variety defined over an algebraically closed field $k$ of arbitrary characteristic, and let $D$ be an $\mathbb{R}$-divisor on $X$ having an $\mathbb{R}-C K M$ Zariski decomposition $D=P+N$.

Then $\mathbf{B}_{+}(D)=\mathbf{B}_{+}(P)$ and $\operatorname{Supp}(N) \subseteq \mathbf{B}_{+}(D)$.

Proof. We will use some results in [N; BBP; ELMNP], and [P]. We point out that, even though in these references, the results mentioned further are proved for smooth varieties over the complex numbers, the results hold with minor modifications on a normal $\mathbb{Q}$-factorial projective variety defined over an algebraically closed field.

If $D$ is not big, then $P$ is also not big, so that $\mathbf{B}_{+}(D)=\mathbf{B}_{+}(P)=X$.

Suppose now that $D$ is big, so that $P$ is also big by [N, Thm. II.3.7 and Lemma II.3.16]. Given any prime divisor $\Gamma$ on $X$, we can define, as in [N, Def. III.1.1],

$$
\sigma_{\Gamma}(D)=\inf \left\{\operatorname{ord}_{\Gamma}(E), E \text { effective } \mathbb{R} \text {-divisor on } X \text { such that } E \equiv D\right\}
$$

and, for any pseudoeffective $\mathbb{R}$-divisor $F$ on $X$, as in [N, Def. III.1.6],

$$
\sigma_{\Gamma}(F)=\lim _{\varepsilon \rightarrow 0^{+}} \sigma_{\Gamma}(F+\varepsilon A),
$$

where $A$ is an ample divisor (the definition does not depend on the choice of $A$ ). Now set

$$
N_{\sigma}(D)=\sum_{\Gamma} \sigma_{\Gamma}(D) \Gamma, \quad P_{\sigma}(D)=D-N_{\sigma}(D) .
$$

Note that $N_{\sigma}(D)$ is an $\mathbb{R}$-divisor by [N, Cor. III.1.11]. The decomposition $D=$ $P_{\sigma}(D)+N_{\sigma}(D)$ is called the $\sigma$-decomposition of $D$ (see [N, Def. III.1.12]). By [N, Rmk. III.1.17(3)] or [P, Rmk. 7.2 and Prop. 4.18] it follows that $P=$ $P_{\sigma}(D)$ and $N=N_{\sigma}(D)$. We also recall that $\operatorname{Supp}\left(N_{\sigma}(D)\right) \subseteq \mathbf{B}(D)$ (see [BBP, Lemma 2.6]). 
Given any ample $\mathbb{R}$-divisor $A$ on $X$ such that $A \leq D$, we find, by [N, Lemma III.1.4], that $\sigma_{\Gamma}(D) \leq \sigma_{\Gamma}(D-A)+\sigma_{\Gamma}(A)=\sigma_{\Gamma}(D-A) \leq \operatorname{ord}_{\Gamma}(D-A)$, whence $D-A \geq N$. Therefore,

$$
\begin{aligned}
\mathbf{B}_{+}(D) & =\bigcap_{A \leq D} \operatorname{Supp}(D-A)=\bigcap_{A \leq D}(\operatorname{Supp}(D-A-N) \cup \operatorname{Supp}(N)) \\
& =\operatorname{Supp}(N) \cup \bigcap_{A \leq P} \operatorname{Supp}(P-A)=\operatorname{Supp}(N) \cup \mathbf{B}_{+}(P) .
\end{aligned}
$$

Now let $\Gamma$ be a prime divisor in the support of $N$, so that $\sigma_{\Gamma}(D)>0$. We will prove that $\Gamma \subseteq \mathbf{B}_{+}(P)$. Let $H$ be an ample Cartier divisor such that $H-\Gamma$ is ample. Then there exists $\varepsilon>0$ sufficiently small such that $\varepsilon \leq \sigma_{\Gamma}(D), \mathbf{B}_{+}(P)=$ $\mathbf{B}(P-\varepsilon(H-\Gamma))$ by [ELMNP, Prop. 1.5] (note that it is not needed that $P-$ $\varepsilon(H-\Gamma)$ is a $\mathbb{Q}$-divisor), and $P-\varepsilon H$ is big. By [N, Lemmas III.1.8 and III.1.4] we get

$$
0<\varepsilon=\sigma_{\Gamma}(P+\varepsilon \Gamma) \leq \sigma_{\Gamma}(P-\varepsilon(H-\Gamma))+\sigma_{\Gamma}(\varepsilon H)=\sigma_{\Gamma}(P-\varepsilon(H-\Gamma)),
$$

so that $\Gamma \subseteq \mathbf{B}(P-\varepsilon(H-\Gamma))=\mathbf{B}_{+}(P)$.

\section{Proofs of the Main Results}

Proof of Theorem 1. We begin by recalling some results of Moriwaki [M]. In [M, Lemma 4.1], ${ }^{2}$ Moriwaki showed that there exist integral curves $C, C_{0}, \ldots, C_{\lfloor g / 2\rfloor}$ inside $\bar{M}_{g}$, not entirely contained in the boundary $\partial \bar{M}_{g}$, with the following properties:

- $C$ is contained inside $M_{g}$;

- $C_{0}$ is contained in $\bar{H}_{g}$ and intersects $\partial \bar{M}_{g}$ in points corresponding to isomorphism classes of irreducible curves with a single node;

- For every $i=1, \ldots,\lfloor g / 2\rfloor, C_{i}$ is contained in $\bar{H}_{g}$ and intersects $\partial \bar{M}_{g}$ in points corresponding to isomorphism classes of stable curves formed by two irreducible components of genus $i$ and $g-i$ meeting in a single node.

It follows from the proof of [M, Prop. 4.2] that the cone spanned by $C, C_{0}, \ldots, C_{\lfloor g / 2\rfloor}$ inside $N_{1}\left(\bar{M}_{g}\right)_{\mathbb{R}}$ is the dual of the cone of M-divisors.

Consider now an $\mathbb{R}$-divisor $D$ on $\bar{M}_{g}$ such that $\mathbf{B}_{-}(D) \subseteq \partial \bar{M}_{g}$ (respectively $\left.\mathbf{B}_{+}(D) \subseteq \partial \bar{M}_{g}\right)$ and let $\gamma$ be one of the curves $C, C_{0}, \ldots, C_{\lfloor g / 2\rfloor}$. Since $\gamma \nsubseteq$ $\partial \bar{M}_{g}$, we get that $\gamma \nsubseteq \mathbf{B}_{-}(D)$ (respectively $\gamma \nsubseteq \mathbf{B}_{+}(D)$ ), and therefore $D \cdot \gamma \geq 0$ (respectively $D_{\mid \gamma}$ is big, that is, $D \cdot \gamma>0$ ). This shows that $D$ is an M-divisor (respectively a strict M-divisor).

Vice versa, suppose first that $D$ is an M-divisor. As observed in the Introduction, it follows from (1) that every M-divisor is an effective linear combination of

\footnotetext{
${ }^{2}$ Which works over an algebraically closed field of characteristic different from two (due to the use of double covers).

${ }^{3}$ Which works over an algebraically closed field of arbitrary characteristic using the extension of the result of Cornalba and Harris [CH, Prop. 4.7] to arbitrary characteristic obtained by Yamaki [Y, Thm. 1.7].
} 
the Moriwaki divisor $M$ and the boundary divisors. Hence, there exist $\beta \geq 0$ and an effective $\mathbb{R}$-divisor $E$ on $\bar{M}_{g}$ such that $D=\beta M+E$ and $\operatorname{Supp}(E) \subseteq \partial \bar{M}_{g}$, where $M$ is the Moriwaki divisor as in (2).

We recall that the content of $\left[\mathrm{M}\right.$, Thm. B] is exactly that $\mathbf{B}_{-}(M) \subseteq \partial \bar{M}_{g}$; hence,

$$
\mathbf{B}_{-}(D) \subseteq \mathbf{B}_{-}(M) \cup \operatorname{Supp}(E) \subseteq \partial \bar{M}_{g} .
$$

Moreover, if $D$ is a strict M-divisor, we can choose a sufficiently small ample $\mathbb{R}$ divisor $A$ on $\bar{M}_{g}$ such that $D^{\prime}:=D-2 A$ is still a strict M-divisor and $\mathbf{B}_{+}(D)=$ $\mathbf{B}(D-A)$ by [ELMNP, Prop. 1.5] (note that it is not needed that $D-A$ is a $\mathbb{Q}$-divisor). Then there exist $\beta^{\prime}>0$ and an effective $\mathbb{R}$-divisor $E^{\prime}$ on $\bar{M}_{g}$ such that $D^{\prime}=\beta^{\prime} M+E^{\prime}$ and $\operatorname{Supp}\left(E^{\prime}\right) \subseteq \partial \bar{M}_{g}$. Hence,

$$
\mathbf{B}_{-}\left(D^{\prime}\right) \subseteq \mathbf{B}_{-}(M) \cup \operatorname{Supp}\left(E^{\prime}\right) \subseteq \partial \bar{M}_{g} ;
$$

therefore, also

$$
\mathbf{B}_{+}(D)=\mathbf{B}(D-A)=\mathbf{B}\left(D^{\prime}+A\right) \subseteq \mathbf{B}_{-}\left(D^{\prime}\right) \subseteq \partial \bar{M}_{g} .
$$

We note that, for some divisors, we can compute exactly the augmented base locus.

Proposition 3.1. Let $g \geq 3$, and let $D \sim a \lambda-b_{0} \delta_{0}-\cdots-b_{\lfloor g / 2\rfloor} \delta_{\lfloor g / 2\rfloor}$ be $a$ big $\mathbb{R}$-divisor on $\bar{M}_{g}$ with $b_{i} \leq 0$ for all $i=0, \ldots,\lfloor g / 2\rfloor$. Then $\mathbf{B}_{+}(D)=\partial \bar{M}_{g}$. Moreover, if $D$ is a $\mathbb{Q}$-divisor, then, for $m \gg 0$ sufficiently divisible, $\varphi_{m D}$ is the Torelli morphism to the Satake compactification $\bar{M}_{g}^{S}:=\operatorname{Proj}\left(R\left(\bar{M}_{g}, \lambda\right)\right)$ of $M_{g}$, which is a normal variety.

Proof. Recall that $\lambda$ is semiample, whence, by [La, Thm. 2.1.15 and 2.1.27], we get an algebraic fiber space $\pi=\varphi_{m \lambda}: \bar{M}_{g} \rightarrow \operatorname{Im} \varphi_{m \lambda} \cong \bar{M}_{g}^{S}$ for $m \gg 0$ sufficiently divisible (this is the Torelli morphism to the Satake compactification) and that $\bar{M}_{g}^{S}$ is normal. Moreover, it is well known that $\operatorname{Exc}(\pi)=\partial \bar{M}_{g}$ (see, e.g., [ACG, Chap. XIV, §5]).

Notice that the restriction of $D$ to $M_{g}$ is linearly equivalent to $a \lambda$. Since $D$ is big and $\lambda$ is semiample, this implies that $a>0$. Let $A$ be an ample $\mathbb{Q}$-divisor such that $\lambda=\pi^{*} A$ and set $F=-b_{0} \delta_{0}-\cdots-b_{\lfloor g / 2\rfloor} \delta_{\lfloor g / 2\rfloor}$, so that $F$ is effective and $\pi$-exceptional. Since $\bar{M}_{g}$ and $\bar{M}_{g}^{S}$ are normal and $\pi$ is birational, we can apply [BBP, Prop. 2.3]:

$$
\mathbf{B}_{+}(D)=\mathbf{B}_{+}\left(\pi^{*}(a A)+F\right)=\pi^{-1}\left(\mathbf{B}_{+}(a A)\right) \cup \operatorname{Exc}(\pi)=\operatorname{Exc}(\pi)=\partial \bar{M}_{g} .
$$

Now if $D$ is a $\mathbb{Q}$-divisor and $m \gg 0$ is such that $m D$ and $m a \lambda$ are Cartier, then

$$
H^{0}\left(\bar{M}_{g}, m a \lambda\right) \cong H^{0}\left(\bar{M}_{g}, m(a \lambda+F)\right) \cong H^{0}\left(\bar{M}_{g}, m D\right),
$$

and the last assertion of the proposition follows.

Proof of Corollary 1. By [BCL, Thm. A], given a big $\mathbb{Q}$-divisor $D$ on $\bar{M}_{g}$, we have that there exists $m_{0} \in \mathbb{N}$ such that $\bar{M}_{g}-\mathbf{B}_{+}(D)$ is the largest open subset 
of $\bar{M}_{g}-\mathbf{B}(D)$ where the maps $\varphi_{m m_{0} D}$ are isomorphisms for every $m \in \mathbb{N}$. Using this, Corollary 1 follows from Theorem 1.

Proof of Corollary 2. Note that $K_{\alpha}:=13 \lambda-(2-\alpha) \delta$ is a strict M-divisor if and only if $\alpha>\frac{3 g+8}{8 g+4}$. Then (i) follows from Corollaries 1 and 2.2.

Assume now that $\alpha=\frac{3 g+8}{8 g+4}$. Then, $K_{\alpha}$ is a (nonstrict) M-divisor, and, moreover, it is big since its slope $s\left(K_{\alpha}\right)=8+\frac{4}{g}$ is larger than the one of a BrillNoether divisor if $g+1$ is composite (see [EH, Thm. 1]) or of the Petri divisor if $g$ is even (see [EH, Thm. 2]). Also, we claim that $\mathbf{B}\left(K_{\alpha}\right)=\mathbf{B}_{-}\left(K_{\alpha}\right)$. Let $x \in \mathbf{B}\left(K_{\alpha}\right)$, and let $v$ be any divisorial valuation with center $\{x\}$. By the finite generation of $R\left(\bar{M}_{g}, K_{\alpha}\right)$, as in [ELMNP, Prop. 2.8] or [BBP, §2.2], we have that $v\left(\left\|K_{\alpha}\right\|\right)>0$, whence $x \in \mathbf{B}_{-}\left(K_{\alpha}\right)$, and the claim is proved (see also Remark 3.3). By Theorem 1 we get $\mathbf{B}\left(K_{\alpha}\right)=\mathbf{B}_{-}\left(K_{\alpha}\right) \subseteq \partial \bar{M}_{g}$, whence $f_{\alpha}$ is defined over $M_{g}$.

In order to prove the second statement of (ii), observe that $K_{\alpha}$ is proportional to the Cornalba-Harris divisor $(8 g+4) \lambda-g \delta$ of [CH, Prop. 4.3]. It follows from [CH, Prop. 4.3, Thm. 4.12] (see also [Y, Cor. 1.8] in positive characteristic) that $K_{\alpha}$ intersects in zero the curves constructed by Cornalba and Harris [CH, p. 469]: ${ }^{4}$ these are curves in $\bar{H}_{g}$ given by a family $\pi: X \rightarrow T$ of stable hyperelliptic curves over a smooth projective curve $T$ obtained as a double cover $\eta: X \rightarrow T \times \mathbb{P}^{1}$ branched over a general curve $B \subset T \times \mathbb{P}^{1}$ of class $(2 g+2,2 m)$ for some $m \geq 1$. Since the image of $T \rightarrow \bar{H}_{g}$ passes through the general point of $\bar{H}_{g}$, it follows that the map $f_{\alpha}$ contracts the hyperelliptic locus $\bar{H}_{g} \subset \bar{M}_{g}$. This finishes the proof of (ii).

Assume finally that $\alpha<\frac{3 g+8}{8 g+4}$. Then $K_{\alpha}$ intersects negatively the CornalbaHarris curves considered, which therefore must belong to $\mathbf{B}_{-}\left(K_{\alpha}\right)$. By what we said before, we deduce that $\bar{H}_{g} \subset \mathbf{B}_{-}\left(K_{\alpha}\right)$, which proves (iii).

In order to prove Corollary 3 , we need the following:

Lemma 3.2. Let $g \geq 3$, and let $D$ be a nonzero $\mathbb{R}$-divisor on $\bar{M}_{g}$. If $D$ is nef, then $D$ is a strict M-divisor.

Proof. If $D \sim a \lambda-b_{0} \delta_{0}-\cdots-b_{\lfloor g / 2\rfloor} \delta_{\lfloor g / 2\rfloor}$ is nef, then by intersecting $D$ with $F$-curves we find that its coefficients must satisfy the following relations (and many others; see [GKM, Thm. 2.1]):

$$
a \geq 12 b_{0}-b_{1} \quad \text { and } \quad 2 b_{0} \geq b_{i} \geq 0 \quad \text { for any } 1 \leq i \leq g / 2 .
$$

From these relations we get the chain of inequalities (for any $1 \leq i \leq g / 2$ )

$$
a \geq 12 b_{0}-b_{1} \geq 10 b_{0} \geq 5 b_{i} .
$$

\footnotetext{
${ }^{4}$ Indeed, it is easily checked, by [CH, Prop. 4.7] (see also [Y, Thm. 1.7] in positive characteristic), that these curves are all numerically proportional to the curve $C_{0}$ constructed in [M, Lemma 4.1].
} 
Now we conclude that the strict Moriwaki inequalities (1) hold for $D$ since (for any $1 \leq i \leq g / 2$ and $g \geq 3$ ) we have that

$$
\begin{aligned}
a & \geq 10 b_{0} \geq 0 \quad \text { with equalities if and only if } a=b_{0}=0, \\
10 b_{0} & \geq \frac{8 g+4}{g} b_{0} \quad \text { with equality if and only if } b_{0}=0, \\
5 b_{i} & \geq \frac{2 g+1}{i(g-i)} b_{i} \quad \text { with equality if and only if } b_{i}=0 .
\end{aligned}
$$

Proof of Corollary 3. Suppose that $D=P+N$ is an $\mathbb{R}-C K M$ Zariski decomposition. Then $P$ is nef and nontrivial because $\kappa(P)=\kappa(D) \geq 1$, whence it is a strict M-divisor by Lemma 3.2. Therefore, by Lemma 2.3 and Theorem 1 we have $\mathbf{B}_{+}(D)=\mathbf{B}_{+}(P) \subseteq \partial \bar{M}_{g}$, so that $D$ is a strict M-divisor again by Theorem 1 . To conclude we just note that

$$
K_{\bar{M}_{g}}=13 \lambda-2 \delta_{0}-3 \delta_{1}-2 \delta_{2}-\cdots-2 \delta_{\lfloor g / 2\rfloor}
$$

is not an M-divisor.

Proof of Corollary 4. Let $a \in \mathbb{N}$ be such that $a K_{\bar{M}_{g}}$ and $a K_{X}$ are Cartier. Now the nonpositivity of $f$ means that we have

$$
a K_{\bar{M}_{g}}=f^{*}\left(a K_{X}\right)+E
$$

with $E \geq 0$ and $f$-exceptional. Setting $P=f^{*}\left(a K_{X}\right)$ and $N=E$, we see immediately that (8) is an $\mathbb{R}-C K M$ Zariski decomposition of $a K_{\bar{M}_{g}}$, thus contradicting Corollary 3. To conclude the proof, recall that, as discussed in the Introduction (after Corollary 3 ), if $K_{\bar{M}_{g}}$ is big, then $\bar{M}_{g}$ has a minimal model $\left(\bar{M}_{g}\right)_{\text {min }}$. Hence, $\left(\bar{M}_{g}\right)_{\text {min }}$ has normal $\mathbb{Q}$-factorial dlt [KM, Def. 2.37] singularities, $K_{\left(\bar{M}_{g}\right)_{\min }}$ is nef, and there is a projective birational map $f: \bar{M}_{g} \rightarrow\left(\bar{M}_{g}\right)_{\min }$ that is $K_{\bar{M}_{g}}$-negative (in fact, $f$ is obtained via contractions and flips of $K_{\bar{M}_{g}}$-negative extremal rays). Then $f$ cannot be a morphism by what we proved before.

REMARK 3.3. It follows from [BBP, Thm. A] that, whenever $K_{\alpha}=13 \lambda-(2-\alpha) \delta$ or $K_{\bar{M}_{g}}$ is big, then $\mathbf{B}_{-}\left(K_{\alpha}\right)=\mathbf{B}\left(K_{\alpha}\right), \mathbf{B}_{-}\left(K_{\bar{M}_{g}}\right)=\mathbf{B}\left(K_{\bar{M}_{g}}\right)$, and every irreducible component of them and of $\mathbf{B}_{+}\left(K_{\alpha}\right), \mathbf{B}_{+}\left(K_{\bar{M}_{g}}\right)$ is uniruled.

\subsection{Characteristic Zero versus Positive Characteristic}

Even though we worked, throughout the paper, over an algebraically closed field of characteristic zero, we believe that all our results can be extended to a field of arbitrary characteristic. For the benefit of the reader, let us specify what is missing in positive characteristic.

(i) The proof of Theorem 1 uses in a crucial way [M, Thm. B], which is currently known only in characteristic zero. The missing ingredient in positive characteristic is, given a smooth projective curve $C$ of genus $g \geq 3$, the validity of 
$\left[\mathrm{M}\right.$, Claim 2.5.1] for the vector bundle $M_{\omega_{C}}:=\operatorname{ker}\left(H^{0}\left(C, \omega_{C}\right) \otimes \mathcal{O}_{C} \stackrel{\mathrm{ev}}{\longrightarrow}\right.$ $\left.\omega_{C}\right)$, namely that there exists an ample line bundle $A$ such that

$$
H^{0}\left(C, \operatorname{Sym}^{m}\left(\operatorname{End}\left(M_{\omega_{C}}\right)\right) \otimes A\right)=0 \quad \text { for every } m \gg 0 .
$$

The semistability of the vector bundles $\operatorname{Sym}^{m}\left(\operatorname{End}\left(\omega_{C}\right)\right)$, which would imply the vanishing in (9), is not known in positive characteristic. Note that $M_{\omega_{C}}$ is semistable (of slope -2 ) in every characteristic (see [PR] or also [EL, Prop. 3.2], whose proof works verbatim for $M_{\omega_{C}}$ ), but this implies the semistability of $\operatorname{Sym}^{m}\left(\operatorname{End}\left(\omega_{C}\right)\right)$ only in characteristic zero. On the other hand, the stronger condition of being strongly semistable (which is preserved by tensor products and symmetric products even in positive characteristic by [M, Thm. 7.2, Cor. 7.3]) fails in positive characteristic for $M_{\omega_{C}}$ for some smooth plane quartics $C$, as it follows by combining [T, Cor. 4.16] and the several examples worked out in [Mo].

(ii) Once the vanishing in (9) has been established, our Theorem 1 would follow in any characteristic different from two (in characteristic two, we would also need to extend the construction of the curves $C_{0}, \ldots, C_{[g / 2]}$ in $[\mathrm{M}$, Lemma 4.1]). From this, Corollaries 3 and 4, and Proposition 3.1 it would also follow in any characteristic different from two. On the other hand, in order to extend Corollaries 1,2, and 2.2 and Remark 3.3 to positive characteristic, we would also need to establish the finite generation of the section ring of the divisor $13 \lambda-(2-\alpha) \delta$ (for $\alpha \in[0,1] \cap \mathbb{Q}$ ) on $\bar{M}_{g}$, which is currently known only in characteristic zero due to [BCHM].

ACKNOWLEDGMENTS. The present collaboration started during the working group "On the Birational geometry of the moduli space of curves" held during the spring semester of the academic year 2011/2012 at the University of Roma Tre. We thank all the participants to the working group for the stimulating atmosphere. We thank C. Camere for pointing out the paper [T]. The third author heard about Hassett's question (see Corollary 2) at the AIM workshop "The log minimal model program for the moduli space of curves", Palo Alto (California, USA), 10-14 December 2012. He would like to thank the organizers J. Alper, M. Fedorchuk, B. Hassett, and D. Smyth for the invitation to participate in the workshop.

\section{References}

[ACG] E. Arbarello, M. Cornalba, and P. A. Griffiths, Geometry of algebraic curves. Volume II, Grundlehren Math. Wiss., 268, Springer, Heidelberg, 2011. With a contribution by Joseph Daniel Harris.

[BCHM] C. Birkar, P. Cascini, C. Hacon, and M. Kernan, Existence of minimal models for varieties of log general type, Amer. J. Math. 23 (2010), no. 2, 405-468.

[BBP] S. Boucksom, A. Broustet, and G. Pacienza, Uniruledness of stable base loci of adjoint linear systems via Mori theory, Math. Z. 275 (2013), no. 1-2, 499-507. 
[BCL] S. Boucksom, S. Cacciola, and A. F. Lopez, Augmented base loci and restricted volumes of divisors on normal varieties, Math. Z. 278 (2014), no. 3-4, 979985.

[CL] S. Cacciola and A. F. Lopez, Nakamaye's theorem on log canonical pairs, Ann. Inst. Fourier (Grenoble) 64 (2014), no. 6, 2283-2298.

[CH] M. Cornalba and J. Harris, Divisor classes associated to families of stable varieties, with applications to the moduli space of curves, Ann. Sci. Éc. Norm. Supér. (4) 21 (1988), no. 3, 455-475.

[EL] L. Ein and R. Lazarsfeld, Stability and restrictions of Picard bundles, with an application to the normal bundles of elliptic curves, Complex projective geometry (Trieste, 1989/Bergen 1989), London Math. Soc. Lecture Note Ser., 179, pp. 149-156, Cambridge Univ. Press, Cambridge, 1992.

[ELMNP] L. Ein, R. Lazarsfeld, M. Mustaţă, M. Nakamaye, and M. Popa, Asymptotic invariants of base loci, Ann. Inst. Fourier (Grenoble) 56 (2006), no. 6, 17011734.

[EH] D. Eisenbud and J. Harris, The Kodaira dimension of the moduli space of curves of genus $\geq 23$, Invent. Math. 90 (1987), no. 2, 359-387.

[F] G. Farkas, Birational aspects of the geometry of $\bar{M}_{g}$, Surveys in differential geometry. Vol. XIV. Geometry of Riemann surfaces and their moduli spaces, Surv. Differ. Geom., 14, pp. 57-110, Int. Press, Somerville, MA, 2009.

[GKM] A. Gibney, S. Keel, and I. Morrison, Towards the ample cone of $\bar{M}_{g, n}$, J. Amer. Math. Soc. 15 (2002), no. 2, 273-294.

[EGA2] A. Grothendieck, Eléments de géométrie algébrique. II. Étude globale élémentaire de quelques classes de morphismes, Inst. Hautes Études Sci. Publ. Math. 8 (1961).

[H] R. Hain, Normal functions and the geometry of moduli spaces of curves, Handbook of moduli. Vol. I, Adv. Lect. Math. (ALM), 24, pp. 527-578, Int. Press, Somerville, MA, 2013.

[HR] R. Hain and D. Reed, On the Arakelov geometry of moduli spaces of curves, J. Differential Geom. 67 (2004), no. 2, 195-228.

[HMo] J. Harris and I. Morrison, Slopes of effective divisors on the moduli space of stable curves, Invent. Math. 99 (1990), no. 2, 321-355.

$[\mathrm{HMu}] \mathrm{J}$. Harris and D. Mumford, On the Kodaira dimension of the moduli space of curves, Invent. Math. 67 (1982), no. 1, 23-88. With an appendix by William Fulton.

[HH1] B. Hassett and D. Hyeon, Log canonical models for the moduli space of curves: the first divisorial contraction, Trans. Amer. Math. Soc. 361 (2009), no. 8, 4471-4489.

[HH2] Log minimal model program for the moduli space of stable curves: the first flip, Ann. of Math. (2) 177 (2013), no. 3, 911-968.

[HK] Y. Hu and S. Keel, Mori dream spaces and GIT, Michigan Math. J. 48 (2000), 331-348.

[KM] J. Kollar and S. Mori, Birational geometry of algebraic varieties, Cambridge Tracts in Math., 134, Cambridge University Press, Cambridge, 1998.

[La] R. Lazarsfeld, Positivity in algebraic geometry, I, Ergeb. Math. Grenzgeb. (3), 48, Springer-Verlag, Berlin, 2004.

[Lo] A. F. Lopez, The Moriwaki divisor is big, 〈http://ricerca.mat.uniroma3.it/users/ lopez/notes.html .

[Mo] P. Monsky, The Hilbert-Kunz multiplicity of an irreducible trinomial, J. Algebra 304 (2006), no. 2, 1101-1107. 
[M] A. Moriwaki, Relative Bogomolov's inequality and the cone of positive divisors on the moduli space of stable curves, J. Amer. Math. Soc. 11 (1998), no. 3, 569-600.

[Mu D. Mumford, Stability of projective varieties, Enseign. Math. (2) 23 (1977), no. 1-2, 39-110.

[N] N. Nakayama, Zariski-decomposition and abundance, MSJ Mem., 14, Mathematical Society of Japan, Tokyo, 2004.

[PR] K. Paranjape and S. Ramanan, On the canonical ring of a curve, Algebraic geometry and commutative algebra, II, pp. 503-516, Kinokuniya, Tokyo, 1988.

[P] Y. G. Prokhorov, On the Zariski decomposition problem, Tr. Mat. Inst. Steklova 240 (2003), 43-72. Translation in Proc. Steklov Inst. Math. 240 (2003), no. 1, 37-65.

[S] D. I. Smyth, Towards a classification of modular compactifications of $M_{g, n}$, Invent. Math. 192 (2013), no. 2, 459-503.

[ST] The Stacks project, 〈http://stacks.math.columbia.edu/〉.

[T] V. Trivedi, Semistability and Hilbert-Kunz, multiplicities for curves, J. Algebra 284 (2005), no. 2, 627-644.

[Y] K. Yamaki, Cornalba-Harris equality for semistable hyperelliptic curves in positive characteristic, Asian J. Math. 8 (2004), no. 3, 409-426.

S. Cacciola

Dipartimento di Matematica e Fisica

Università di Roma Tre

Largo San Leonardo Murialdo 1

00146, Roma

Italy

cacciola@mat.uniroma3.it

F. Viviani

Dipartimento di Matematica e Fisica

Università di Roma Tre

Largo San Leonardo Murialdo 1

00146, Roma

Italy

\author{
A. F. Lopez \\ Dipartimento di Matematica e Fisica \\ Università di Roma Tre \\ Largo San Leonardo Murialdo 1 \\ 00146, Roma \\ Italy
}

lopez@mat.uniroma3.it

\section{filippo.viviani@gmail.com}

